(c) 2017 International Association for Ecology and Health (outside the USA)

\title{
Phylogenetic Insight into Zika and Emerging Viruses for a Perspective on Potential Hosts
}

\author{
Diana S. Weber, ${ }^{1}$ Karen A. Alroy, ${ }^{2}$ and Samuel M. Scheiner ${ }^{2}$ \\ ${ }^{1}$ S\&T Policy Fellowship, American Association for the Advancement of Science, 1200 New York Avenue NW, Washington, DC 20005 \\ ${ }^{2}$ Division of Environmental Biology, National Science Foundation, 4201 Wilson Blvd., Arlington, VA 22230
}

\begin{abstract}
Global viral diversity is substantial, but viruses that contribute little to the public health burden or to agricultural damage receive minimal attention until a seemingly unimportant virus becomes a threat. The Zika virus (ZIKV) illustrated this, as there was limited information and awareness of the virus when it was identified as a public health emergency in February 2016. Predicting which virus may pose a future threat is difficult. This is in part because significant knowledge gaps in the basic biology and ecology of an emerging virus can impede policy development, delay decision making, and hinder public health action. We suggest using a phylogenetic framework of pathogens and their infected host species for insight into which animals may serve as reservoirs. For example, examining flaviviruses closely related to ZIKV, the phylogenetic framework indicates New World monkeys are the most likely candidates to be potential reservoirs for ZIKV. Secondarily, mammals that are in close proximity to humans should be considered because of the increased opportunity for pathogen exchange. The increase in human-mediated environmental change is accelerating the probability of another previously overlooked virus becoming a significant concern. By investing in basic science research and organizing our knowledge into an evolutionary framework, we will be better prepared to respond to the next emerging infectious disease.
\end{abstract}

Keywords: Flavivirus, Zika, Hosts, Phylogenetic associations, Emerging viruses, Arthropod vectors, Reservoirs

Electronic supplementary material: The online version of this article (doi:10.1007/ s10393-017-1237-x) contains supplementary material, which is available to authorized users.

Disclaimer This work was done by DSW as an activity supported by an AAAS Science \& Technology Policy Fellowship served at the National Science Foundation. This work was done by KAA during off NSF duty time. This work was done by SMS while serving at the US National Science Foundation. The views expressed in this paper do not necessarily reflect those of the National Science Foundation, the United States Government, or the American Association for the Advancement of Science.

Published online: April 18, 2017

Correspondence to: Diana S. Weber, e-mail: diana.weber@gmail.com

\section{INTRODUCTION}

The Zika virus (ZIKV) is just the latest in a growing list of pathogens [e.g., Ebola virus, avian influenza virus, West Nile virus (WNV)] that threaten human health, agricultural resources, and wildlife. Global viral diversity is substantial, but viruses that have a low public health burden or cause negligible agricultural damage tend to receive little to no attention. However, viral evolution, spillover events, or 
changes in hosts can turn seemingly unimportant viruses into biosecurity threats or public health emergencies. Case in point, the scientific community had limited information or awareness of ZIKV when the World Health Organization (WHO) identified it as a public health emergency in February 2016. We know that the emergence of a new pathogen can be caused by changes in human population distributions and behaviors, land use and biodiversity shifts, climate change, international trade, and changes in vector or reservoir host ecology (Woolhouse and GowtageSequeria 2005; Morse et al. 2012; Woolhouse et al. 2014). Beyond identifying likely locations of emerging diseases (Jones et al. 2008), it is difficult to predict the next specific public health crisis and prevent the next pandemic (Han et al. 2016). During an outbreak, public health organizations scramble to understand the nature of a threat and what actions are required to mitigate it. Significant overall knowledge gaps regarding the transmission and maintenance of an emerging virus can delay policy and public health response. Our need to guide policy in the face of incomplete knowledge highlights the importance of basic science research.

When scant information is available about a novel emerging virus, understanding it within a framework of evolutionary relationships can help characterize it in the context of its most closely related species and their known hosts and vectors. Here, we suggest that examining the biology and ecology of related viral species provides predictions about characteristics of emerging understudied viruses. We illustrate that process by presenting a Flavivirus phylogeny case study for ZIKV and its taxonomic relatives, asking within this framework, which animals might serve as reservoirs for ZIKV.

\section{Using Phylogenetic Information}

Evolutionary methods can be used in the fields of public health and epidemiology in two ways. The first use is in tracing the spread of a pathogen. Temporal and geographic pathogen spread can be investigated through molecular epidemiology and evolution, as was done during the severe acute respiratory syndrome coronavirus (SARS-CoV) outbreak (Zhao 2007). At local scales, phylogenetic analyses can be used to identify exposure sources within a small population (Azarian et al. 2015).

The second use of phylogenetic information, and our focus, is to predict relationships among pathogens and their hosts and/or vectors. This information is obtained from two different patterns of relationships: (1) the extent to which closely related pathogens (viruses for this study) share the same hosts or vectors and (2) the extent to which closely related hosts and vectors share the same pathogens. Regarding the later, hosts tend to be phylogenetically related for a given pathogen, though this relationship can vary by pathogen type (Davies and Pedersen 2008); for example, in primates, helminths tend to infect either a single species or members of the same genus, but viruses can infect species across multiple families or orders (Pedersen et al. 2005). Among primates, closely related species (e.g., chimpanzees) are more likely to share pathogens with humans (Davies and Pedersen 2008). Other factors, such as frequency and quantity of exposure, host immunity, and genetic variability of host or pathogen populations, can also inform new host-virus relationships and can influence whether a host is susceptible to a novel pathogen (Davies and Pedersen 2008; Morse et al. 2012).

\section{A Zika Virus Case Study}

It is unknown whether an animal species may become a reservoir host for ZIKV in the Americas and Caribbean. As a reservoir host, a species must contribute to the maintenance and transmission of a pathogen (Haydon et al. 2002). Currently, ZIKV is believed to be transmitted only among humans, though in its native range in Africa, ZIKV is present among non-human Old World primates (Dick et al. 1952). The existence of an animal reservoir may change local and regional control strategies because eliminating the virus from vectors and local human populations does not equate to complete local eradication of the virus. Generally, surveying all domestic and wildlife species for the presence of a virus is cost and time prohibitive. Therefore, combining what is known regarding non-human hosts for ZIKV with similar information from closely related viruses will help prioritize animal surveys.

To that end, we surveyed the flavivirus literature for evidence of host infection, aggregating the results to the taxonomic level of order or above for mammalian, avian, and reptilian hosts (see Figure 1, table S1). With respect to the vectors, the viruses divide into two groups. One group consists of two clades (one comprised of Dengue viruses and the second of ZIKV and Kedougou viruses) that are transmitted by Aedes mosquitoes. The second, larger group consists of viruses that are transmitted by Culex mosquitoes 


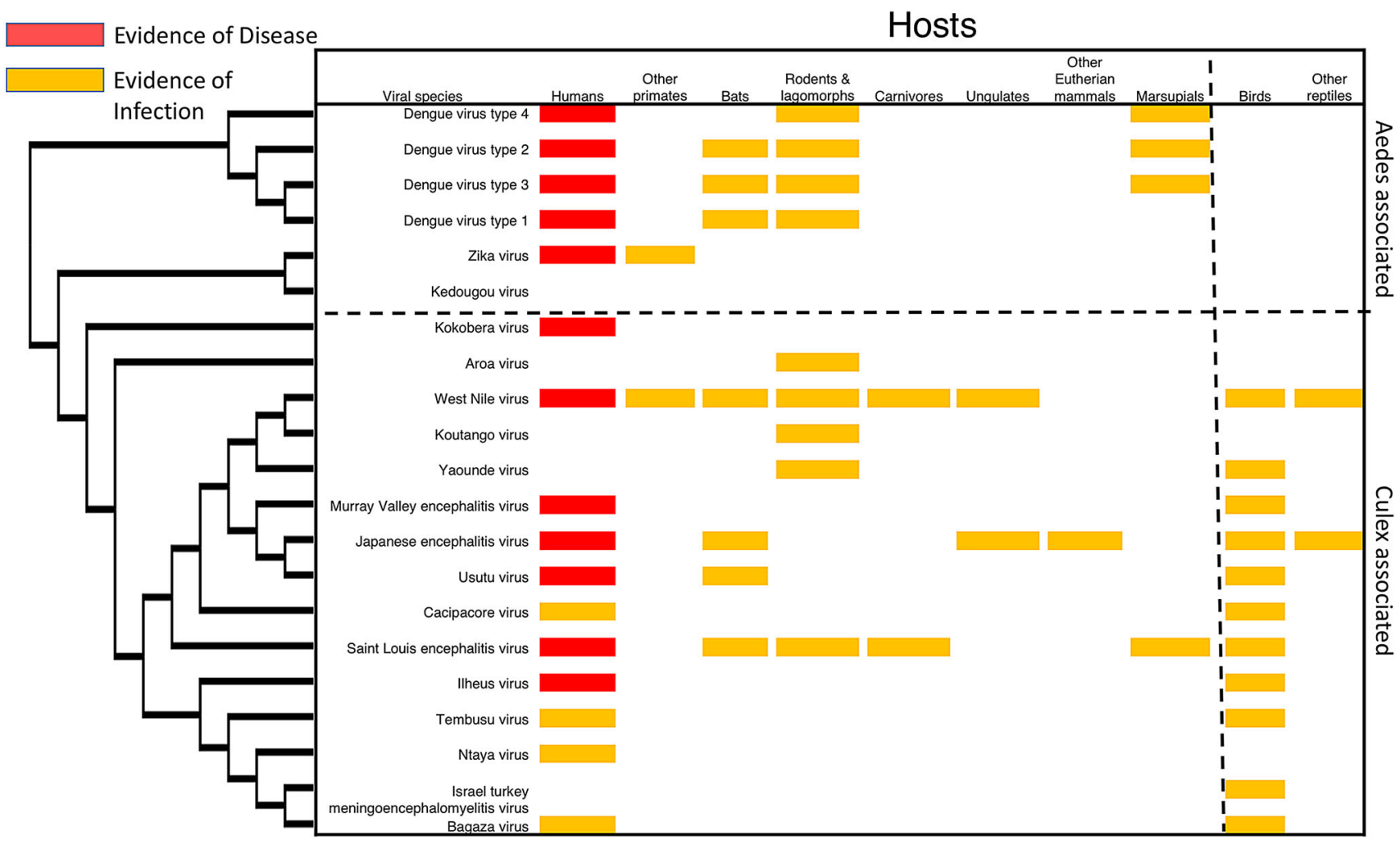

Fig. 1. Patterns of infection among Flavivirus species and their hosts. The diagrammed phylogeny is for part of the Flavivirus genus and only includes those viruses closely related to ZIKV virus. The phylogenetic relationships on the left are based on the published viral phylogeny of Moureau et al. (2015). The figure shows patterns of relationships but not the distance of those relationships. The vertical dashed line to the far-right separates mammalian and non-mammalian hosts; the horizontal dashed line segregates viruses that have Aedes mosquitoes as their vector (above) from those transmitted by Culex species (below). For this figure, we used the viral taxonomy from Pletnev et al. (2011). The data for incidence were obtained by surveying the viral literature for evidence of infection or disease in vertebrate hosts, aggregated by host categories and viral species. Filled cells indicate evidence that one or more species in that group has shown evidence of infection (orange) or disease (red, humans only) by at least one host species in that taxonomic group [Supplemental Table S1]. Evidence of exposure (i.e., the presence of antibodies) was not incorporated into this figure for three reasons: the uncertainty due to potential for cross-reactivity with other flaviviruses, the inability of serologic data to provide definite evidence of infection ( $c f$. Levinson et al. 2013), and the great variability in serologic methods (Color figure online).

[e.g., WNV and Japanese encephalitis virus (JEV)]. This grouping by mosquito vector is associated with host relationships. For the Culex-associated viruses, birds are the major reservoirs likely because Culex mosquitoes prefer to feed on birds. In contrast, for the Aedes-associated viruses, mammals are the major reservoirs because those mosquitoes feed primarily on mammalian hosts. Furthermore, a recent study of ZIKV in Culex suggests that this genus may not be a competent vector for ZIKV (Huang et al. 2016).

These relationships suggest non-human mammals should be considered as possible animal reservoirs for ZIKV in initial animal surveys. New World monkeys are the most likely potential reservoir because of evidence of their infection with ZIKV (Hayes 2009; Favoretto et al. 2016). Secondary consideration should be given to other mammals. Hosts that are in proximity to humans have increased opportunity for the exchange of pathogens. Evidence of exposure (i.e., antibodies) to ZIKV has been documented in various other mammalian species (bats, rodents), including domesticated species (horses, water buffalo, cows, sheep, and goats) (Darwish et al. 1983; Olson et al. 1983). Such associations are consistent with the tendency of arthropod-borne viruses to have a host range that stretches across multiple families or orders (Pedersen et al. 2005). Primates are a reservoir for many human diseases (Davies and Pedersen 2008; Levinson et al. 2013). Therefore, we typically focus on spillover from other primates to humans; however, for ZIKV in the Americas it is the reverse transition that is of concern. Beyond public health considerations, it is unknown how animal health and conservation efforts may be affected by ZIKV. 


\section{Challenges in Viral Phylogenetics}

Using viral phylogenetic information has a number of challenges for all viruses and specifically for flaviviruses. Most viruses and host species have not been uniformly and comprehensively surveyed, and often the surveys tend to be biased toward viruses that affect humans or livestock. Viruses exclusively affecting wildlife usually receive less attention (Dick et al. 1952; Han et al. 2016). For example, the host species literature is robust for some flaviviruses (e.g., WNV, JEV) (S1 table), facilitating an understanding of transmission ecology and dynamics, whereas for others, only a single isolate is known (e.g., Iguape virus and Sal Vieja virus) (Moureau et al. 2015).

Viral systematics is still in its infancy compared to that of eukaryotes, bacteria, and archaea. Although enhanced sequencing capabilities can now provide genomic data for phylogeny reconstruction, there are still numerous obstacles that make consensus viral phylogenies elusive. It is difficult to discern distant relationships because viral phylogenies are built on few genes and viruses have high mutation rates. This is particularly true for RNA viruses such as the Flavivirus genus (Ali and Ali 2015; Moureau et al. 2015).

Though the International Committee on Taxonomy of Viruses (ICTV) continually updates viral taxonomy continually, uncertainty exists because of knowledge gaps and debate about what information should be used, how viral species should be distinguished, and what criteria should be applied for the designation of various taxonomic categories (e.g., subtypes, species, families) (Kuhn and Jahrling 2010). Historically, viral taxonomies have been based on biological and physical properties of a virus (e.g., the type of viral genome, the cross-reactivity patterns of antibody sera, transmission characteristics, and vector relationships) (Murphy et al. 2012). New Flavivirus strains and isolates are continually being discovered, and many have yet to be classified making viral taxonomy and phylogenetic reconstruction challenging at best (Moureau et al. 2015).

\section{CONCLUSION}

A greater investment in viral systematics can help to ameliorate these challenges. Beyond the viral phylogenies themselves, these frameworks can be used to compile and organize the literature concerning the biology and ecology of pathogens and their related hosts and vectors; such compilations are beginning. Levinson et al. (2013) developed a database of mammalian hosts and associated viruses to determine a surveillance strategy for identifying novel diseases. Databases, such as GenBank, the Virus Pathogen Resource (ViPR), and the Influenza Research Database (IRD), partially achieve the goal of linking sequence data with host associations, known vectors, geographic distributions, and clinical information. However, no single database integrates all this information. Such linkages, combined with examining the literature in a phylogenetic framework, would contribute to the understanding of a pathogen during an outbreak, indicate knowledge gaps, and help prioritize research needs. With their methodology, Anthony et al. (2013) estimated the potential number of undiscovered diseases and compared this in hosts that are geographically and ecologically different. Other researchers have used phylogenetic frameworks in the context of pathogen predication; some have focused on identifying taxonomic host groups with pathogens most likely to spillover to humans as emerging zoonotic diseases (Han et al. 2016). These authors emphasize the need to use phylogenetic frameworks during outbreaks when knowledge of a particular emerging pathogen is limited. The increasing global viral diversity (Jones et al. 2008) makes it difficult to predict which one may pose a future threat to human health, livestock, and wildlife populations. As global environmental change continues to escalate (e.g., land use change, climate change, human migration, and travel), there is an increasing probability that another previously overlooked virus could become a pathogen of significant concern. By investing in basic science research and organizing our knowledge into an evolutionary framework, we will be better prepared to respond to the next emerging infectious disease.

\section{ACKNOWLEDGMENTS}

We thank Ernest Gould for the extensive time he took to provide guidance and insight with this paper. We additionally thank Gordon Burleigh, Susan Perkins, and Susanna Remold for providing thoughtful comments. We thank the NSF library for all their support during this endeavor and especially the NSF librarians, Maura Mullins, and Brock Temanson without whom we could not have accomplished this exhausted literature search. 


\section{REFERENCES}

Ali A, Ali I (2015) The complete genome phylogeny of geographically distinct dengue virus serotype 2 isolates (1944-2013) supports further groupings within the cosmopolitan genotype. PLoS ONE 10(9):e0138900. doi:10.1371/journal.pone.0138900

Anthony SJ, Epstein JH, Murray KA, Navarrete-Macias I, Zambrana-Torrelio CM, Solovyov A, Ojeda-Flores R, Arrigo NC, Islam A, Ali Khan S, Hosseini P, Bogich TL, Olival KJ, SanchezLeon MD, Karesh WB, Goldstein T, Luby SP, Morse SS, Mazet JAK, Daszak P, Lipkin WI (2013) A strategy to estimate unknown viral diversity in mammals. mBio 4(5): e00598-13. doi:10.1128/mBio.00598-13.

Azarian T, Cook RL, Johnson JA, Guzman N, McCarter YS, Gomez N, Rathore MH, Morris JG, Salemi M (2015) Wholegenome sequencing for outbreak investigations of methicillinresistant Staphylococcus aureus in the neonatal intensive care unit: time for routine practice? Infection Control \& Hospital Epidemiology 36:777-785

Darwish MA, Hoogstraal H, Roberts TJ, Ahmed IP, Omar F (1983) A sero-epidemiological survey for certain arboviruses (Togaviridae) in Pakistan. Transactions of the Royal Society of Tropical Medicine and Hygiene 77:442-445

Davies TJ, Pedersen AB (2008) Phylogeny and geography predict pathogen community similarity in wild primates and humans. Proceedings of the Royal Society B: Biological Sciences 275:1695-1701

Dick GWA, Kitchen SF, Haddow AJ (1952) Zika virus. I. Isolations and serological specificity. Transactions of the Royal Society of Tropical Medicine and Hygiene 46:509-520

Favoretto S, Araujo D, Oliveira D, Duarte N, Mesquita F, Zanotto P, Durigon E (2016) First detection of Zika virus in neotropical primates in Brazil: a possible new reservoir. bioRxiv. Available: http://dx.doi.org/10.1101/049395. [accessed October 19, 2016]

Han BA, Kramer AM, Drake JM (2016) Global patterns of zoonotic disease in mammals. Trends in Parasitology 32:565-577

Haydon DT, Cleaveland S, Taylor LH, Laurenson MK (2002) Identifying reservoirs of infection: a conceptual and practical challenge. Emerging Infectious Diseases 8:1468-1473

Hayes EB (2009) Zika virus outside Africa. Emerging Infectious Diseases 15:1347-1350

Huang YJS, Ayers VB, Lyons AC, Unlu I, Alto BW, Cohnstaedt LW, Higgs S, Vanlandingham DL (2016) Culex species mosquitoes and Zika virus. Vector-Borne and Zoonotic Diseases $16: 673-676$

Jones KE, Patel NG, Levy MA, Storeygard A, Balk D, Gittleman JL, Daszak P (2008) Global trends in emerging infectious diseases. Nature 451:990-994. doi:10.1038/nature06536
Kuhn JH, Jahrling PB (2010) Clarification and guidance on the proper usage of virus and virus species names. Archives of Virology 155:445-453

Levinson J, Bogich TL, Olival KJ, Epstein JH, Johnson CK, Karesh W, Daszak P (2013) Targeting surveillance for zoonotic virus discovery. Emerg Infect Dis 19:743-747. doi:10.3201/eid1905. 121042

Morse SS, Mazet JA, Woolhourse M, Parrish CR, Carroll D, Karesh WB, Zambrana-Torrelio C, Lipkin WI, Daszak P (2012) Prediction and prevention of the next pandemic zoonosis. Lancet 380:1956-1965

Moureau G, Cook S, Lemey P, Nougairede A, Forrester NL, Khasnatinov M, Charrel RN, Firth AE, Gould EA, de Lamballerie X (2015) New insights into Flavivirus evolution, taxonomy and biogeographic history, extended by analysis of canonical and alternative coding sequences. PLoS ONE 10:e117849

Murphy FAIn: Fauquet CMBishop DHGhabrial SAJarvis AMartelli GPMayo MASummers MD (editors) (2012) Virus Taxonomy: Classification and Nomenclature of Viruses, Wein: Springer

Olson JG, Ksiazek TG, Gubler DJ, Lubis SI, Simanjuntak G, Lee VH, Nalim S, Juslis K, See R (1983) A survey for arboviral antibodies in sera of humans and animals in Lombok, Republic of Indonesia. Annals of Tropical Medicine and Parasitology 77:131-137

Pedersen AB, Altizer S, Poss M, Cunningham AA, Nunn CL (2005) Patterns of host specificity and transmission among parasites of wild primates. International Journal for Parasitology 35:647-657

Pletnev A, Gould E, Heiz FX, Meyers G, Thiel HJ, Bukh J, Stiasny K, Collett MS, Becher P, Simmonds P, Rice CM, Monath TP (2011) Flaviviridae. In: Virus Taxonomy, King AMQ, Adams MJ, Carstens EB, Lefkowitz EJ (editors), Oxford: Elsevier, pp 10031020

Woolhouse MEJ, Gowtage-Sequeria S (2005) Host range and emerging and reemerging pathogens. Emerging Infectious Diseases 11:1842-1847

Woolhouse MEJ, Adair K, Brierley L (2014) RNA viruses: a case study of the biology of emerging infectious diseases. Microbiology Spectrum 1(1):OH-0001-2012. doi:10.1128/microbiolspec. OH-0001-2012 [Online: November 7, 2016]

Zhao GP (2007) SARS molecular epidemiology: a Chinese fairy tale of controlling an emerging zoonotic disease in the genomics era. Philosophical Transactions of the Royal Society B: Biological Science 362:1063-1081 